\title{
VIGILANCIA EPIDEMIOLÓGICA EN RIESGO OSTEOMUSCULAR PARA OCHO (8) LÍNEAS PRODUCTIVAS EN UNA INDUSTRIA DE ALIMENTOS BOGOTA-COLOMBIA
}

\author{
Epidemiological Surveillance In Musculoskeletal Risk For Eight (8) Production Line \\ In A Food Industry Bogota-Colombia
}

\author{
Flor Esperanza Rodriguez Ferro (1) \\ Carrillo, Ginna (2); Roa, Angélica (3); Sarmiento, Angélica (4) .
}

\begin{abstract}
Resumen
Este artículo, expone los resultados de un proceso de investigación formativa, llevado a cabo durante 864 horas, por tres estudiantes de Terapia Ocupacional guiados académicamente, que contó con el consentimiento informado de los involucrados. Se parte de la fundamentación disciplinar para intervenir en empresas como escenario de acción profesional, así como de las relaciones que se establecen con salud ocupacional y ergonomía. La metodología utilizada fue la del marco lógico, con la evaluación y diagnostico de las condiciones de salud de los trabajadores y de los puestos de trabajo en 8 líneas de producción. Los instrumentos utilizados, fueron: 1) matriz socio Demográfica, 2) auto reporte de condiciones de salud, 3) análisis ocupacional y 4) herramienta ergonómica OWAS. Como principales resultados, se realizó la caracterización de: población, factores de riesgo osteo muscular, y categorización de los puestos de trabajo por nivel de riesgo. Como conclusión, logró aplicarse las recomendaciones emitidas en la empresa, garantizándose el cumplimiento de los indicadores establecidos para favorecer el mejor desempeño ocupacional de la población, desde la proyección de Terapia Ocupacional como una profesión que aplica sus conocimientos en el bienestar de los trabajadores y en el estudio de la naturaleza del desempeño ocupacional de la persona y los contextos.
\end{abstract}

Palabras claves: Terapia Ocupacional, sector trabajo, empresa, marco lógico, evaluación, riesgo osteo muscular

\begin{abstract}
This article describes the results of a process of formative research, conducted over 864 hours, by three students of Occupational Therapy, teaching responsibility under, with the informed consent of those involved. It is part of the grounds for discipline as companies involved in professional theater of action, as well as the relations established with occupational health and ergonomics. The methodology used was the logical framework, the assessment and diagnosis of health conditions of workers and jobs in 8 lines. The instruments used were: 1) sociodemographic matrix, 2) self-report of health conditions, 3) occupational analysis and 4) ergonomic tool OWAS. As main results, we conducted the characterization of population, risk factors for osteo-muscular, and categorization of jobs by level of risk. In conclusion, we succeeded in implementing the recommendations made in the company, ensuring compliance with the indicators established to promote the occupational performance of people, from the projection of Occupational Therapy as a profession that applies its expertise in the welfare of workers and the study of the nature of the occupational performance of individuals and contexts
\end{abstract}

Key words: Occupational Therapy, sector work, enterprise, logical framework, assessment, risk osteo muscular

1. Terapeuta Ocupacional. ASO. Magíster en Discapacidad e Inclusión Social. Profesora asesora pasantía. Profesora Programa de Terapia Ocupacional. Facultad de Medicina y Ciencias de la Salud. Universidad del Rosario. Contacto: flor.rodriguez@urosario.edu.co. Dirección: Cra 24 No.63 C-69. Bogota-Colombia

2. Terapeuta Ocupacional en formación. Contacto: ginapaola192@yahoo.es

3. Terapeuta Ocupacional en formación. Contacto: ansarcar@hotmail.com

4. Terapeuta Ocupacional en formación. Contacto: angieroa85@hotmail.com 


\section{Introducción}

En la actualidad, el bienestar del trabajador se relaciona directamente con factores tales como la productividad y los sistemas de gestión y calidad integral que consideran las condiciones de salud como una variable de intervención constante por parte de los programas de Salud Ocupacional de las empresas; a estas acciones hoy en día se les conoce como "Retribución Emocional"1. Las mismas, se encaminan a que el trabajador, no solo reciba una remuneración económica, sino, servicios ofrecidos por la empresa que se centran en el mantenimiento físico y psicológico de sus empleados.

Relacionado con lo anterior, Terapia Ocupacional, ha incursionado en el sector trabajo, elaborando e implementando diversos programas, que se centran en proporcionar bienestar, tanto físico como psicosocial al trabajador, su ambiente y la relación que establece con este, así como en los factores que pueden influir asertivamente o negativamente en la ejecución de su ocupación.

Las empresas que buscan la implementación de estos programas centran sus objetivos en una mayor productividad en entornos saludables, así como en la prevención de accidentes o enfermedades profesionales, mediante el análisis de puestos de trabajo, realización de análisis ocupacionales y uso de herramientas ergonómicas, que llegan hasta reubicaciones laborales o modificación del puesto de trabajo.

Es así como la industria de alimentos, en la cual se gestiono el proyecto aquí socializado ha venido buscando ese bienestar físico y psicosocial de sus empleados; por esta razón, ha dispuesto de espacios y materiales necesarios, para que estudiantes del programa de Terapia Ocupacional de la Universidad del Rosario implementen un Sistema de Vigilancia Epidemiológica en Factores de riesgo Osteomusculares. La aplicación del mismo, se desarrolló mediante el planteamiento y resolución de dos necesidades; las cuales fueron satisfechas posterior a la aplicación de instrumentos evaluativos utilizados tanto en el trabajador como en su entorno de trabajo, para así establecer un diagnostico y un conjunto de recomendaciones.

\section{Fundamentos teóricos}

En la década de los 80’s, en Colombia los profesionales de Terapia Ocupacional comenzaron a incursionar en el área de Salud Ocupacional, a partir de la instauración del contexto normativo para la organización y administración de programas de Salud Ocupacional mediante decretos ${ }^{2}$; y resoluciones ${ }^{3}$ derivadas del ámbito jurídico así como por los fundamentos teóricos y experiencia de los profesionales en el análisis de puestos de trabajo y en el diseño de sistemas o programas de vigilancia epidemiológica en ergonomía.

La salud ocupacional en Colombia, surgió históricamente antes que la ergonomía, razón por la cual se ha concebido como un área de trabajo interdisciplinario enfocada a la promoción y atención de los trabajadores frente a los riesgos o condiciones de trabajo. Desde los años noventa, la salud ocupacional se rige por las normas del sistema de seguridad social integral $^{4}$ y más específicamente para lo que se considera en Colombia como el Sistema General de Riesgos Profesionales ${ }^{5}$, orientado a las acciones de intervención propias para las personas que trabajan $\mathrm{y}$ se encuentran afiliadas a una administradora de riesgos profesionales, 
-ARP- para efectos de protección ante una enfermedad o accidente relacionado con su empleo.

Actualmente, los escenarios en los cuales están ejerciendo los terapeutas ocupacionales en salud ocupacional son: las empresas, las administradoras de riesgos profesionales (ARP), las instituciones prestadoras de servicios (IPS) en salud ocupacional y el nivel ejecutivo del gobierno. Algunas de las funciones que cumplen estos profesionales son: participación como miembro del comité paritario de salud ocupacional; levantamiento de panorama de riesgos; estudio de puestos de trabajo; liderazgo del programa de salud ocupacional en las empresas; asesoría en el diseño del Plan de Salud Ocupacional, educación a trabajadores; vigilancia y control, precalificación de invalidez; y participación en las juntas de calificación de invalidez. ${ }^{6}$

Con base en lo expuesto aquí, y dada el área en la cual se llevo a cabo este proceso de investigación formativa, mediante la modalidad de pasantía, aspectos construidos desde la academia fueron retomados como aparte del marco referencial que fundamento el producto final. Algunos de estos aspectos, se relacionan específicamente con Terapia Ocupacional en el ámbito o sector laboral, en el cual se incluye la salud ocupacional.

Es decir, debido a los procesos de industrialización y desarrollo que se han dado en las ultimas décadas, donde la automatización esta ganando cada vez más fuerza y por tanto, los trabajadores se han visto obligados a aumentar sus niveles de producción y rendimiento, poniendo en riesgo su salud tanto física como mental, Terapia Ocupacional ha requerido hacer uso de mayores herramientas conceptuales y prácticas que posicionen su desempeño en el sector y den fe de la intervención en el proceso ocupacional denominado trabajo

El papel de Terapia Ocupacional en salud ocupacional ${ }^{7}$ parte de su conocimiento sobre función y disfunción ocupacional, los cuales constituyen el eje del quehacer profesional, independientemente del enfoque que se tome (biologista, social, psicosocial...); diferentes autores citados en el documento guía para la práctica laboral en la Universidad del Rosario, Programa de Terapia Ocupacional definen estos conceptos como:

\section{Función:}

- Fidler y Mosey: En su teoría del desarrollo adaptativo definen este estado como el balance entre las destrezas en términos de eficacia y competencia.

- Wlbarger y Ayres: En su teoría del biodesarrollo lo plantean en términos de destrezas adaptativas de los sistemas sensoriales.

- Really: En la teoría de trabajo y juego lo define como autodirección en el logro de roles.

- Algunos enfoques modernos se basan en la teoría de sistemas y consideran al hombre como un sistema abierto que interactúa con su medio ambiente.

- Kielhofner (1985): considera que una persona es adaptativa (funcional) cuando es capaz de satisfacer los desafíos, expectativas y oportunidades del ambiente.

- Howe y Briggs (1982): en su modelo denominado Sistema Ecológico considera al individuo como un sistema abierto que se influencia mutuamente con su medio ambiente, concibe la función en términos de efectividad y logro de objetivos personales: si el desarrollo de roles y tareas es efectivo en la adquisición de objetivos, se dice que la conducta es funcional. 


\section{Disfunción:}

- Clark Pate (1974): Los estados de disfunción se conciben como imbalances producidos por procesos internos o externos, deterioro para interactuar en el medio ambiente, en la participación y en la adaptación.”8

Por otro lado, la Terapia Ocupacional ha concebido desde sus inicios, la relación existente entre el hombre - puesto de trabajo (ocupación), donde el trabajador es tomado como un sistema abierto en interacción con aspectos biológicos-ambientales del sitio de trabajo, puesto que su función se afecta en la medida en que es expuesto a factores de riesgo de tipo: físico, químico, osteomuscular o musculo esquelético; también reconocidos, en alguna literatura y contexto, como ergonómico; psicosocial y otros que pueden ser causantes de accidentes, enfermedades y/o desmotivación.

A partir de esto, y teniendo en cuenta que el objeto de estudio de Terapia Ocupacional es precisamente la Ocupación Humana, los profesionales de ésta área de la salud han visto la necesidad de intervenir en el ámbito o sector laboral, dado en que en esta es donde el individuo se autorealiza y hace uso al máximo de sus potencialidades para ejecutar roles sociales en un ambiente laboral.

La salud Ocupacional, es entonces una rama de las ciencias de la salud que se encarga de mantener el más alto grado de bienestar físico, mental y social de los trabajadores, mediante la prevención y promoción de hábitos y estilos de vida y trabajo saludable. De igual forma, es definida como un componente de la salud integral, conformada por un conjunto de políticas, estrategias y acciones como los recursos humanos, técnicos y financieros, dirigidos a mejorar la calidad de vida del trabajador y el desarrollo empresarial

A partir de esto, el papel de Terapia Ocupacional en la misma es muy importante al asumir la ocupación humana como su principal objeto de estudio. Por tanto, posee las bases conceptuales necesarias para: promover y mantener los más altos estándares en la función ocupacional y bienestar de los trabajadores, haciendo uso de estrategias que involucran aspectos adaptativos biopsicosociales y espirituales del trabajador, mejorando de esta forma la eficiencia y productividad de la empresa; lo cual se constituye como su principal misión en el ámbito o sector referido.

Así, las funciones del profesional se pueden visualizar en el siguiente esquema:

\begin{tabular}{|c|c|}
\hline & FUNCIONES DEL TERAPEUTA OCUPACIONAL EN EL SECTOR \\
LABORAL
\end{tabular}




\begin{tabular}{|c|c|}
\hline & $\begin{array}{l}\text { FUNCIONES DEL TERAPEUTA OCUPACIONAL EN EL SECTOR } \\
\text { LABORAL }\end{array}$ \\
\hline Administrativas & $\begin{array}{l}\text { - Diseño de perfiles ocupacionales. } \\
\text { - Planeación de sub-programas: inducción, prejubilación, motivación ... } \\
\text { - Coordinación de programas de Salud Ocupacional. } \\
\text { - Toma de decisiones en: selección de personal, ubicación y reubicación laboral. } \\
\text { - Control de acciones preventivas. } \\
\text { - Registro de estadísticas ocupacionales. } \\
\text { LOGROS: Racionalización del recurso y aproximación a la excelencia en el } \\
\text { desempeño. }\end{array}$ \\
\hline Asistenciales & $\begin{array}{l}\text { Promoción y prevención de las disfunciones ocupacionales. } \\
\text { Habilidades y/o rehabilitación de los trabajadores en los aspectos psicosociales, físicos y } \\
\text { sensoperceptuales. } \\
\text { Fortalecimiento de la intervención profesional mediante una estrecha coordinación y } \\
\text { ejecución con el equipo de salud ocupacional. } \\
\text { LOGROS: Disminución de los índices de ausentismo y de reubicación, así como } \\
\text { mejoramiento de las condiciones de trabajo. }\end{array}$ \\
\hline Educativas & $\begin{array}{l}\text { - Diseño, desarrollo y ejecución de programas educativos para la prevención de } \\
\text { accidentes y enfermedades profesionales. } \\
\text { - Diseño, desarrollo y ejecución de programas motivacionales para los trabajadores. } \\
\text { LOGROS: Capacitación y motivación = disminución del índice de ausentismo, bienestar y } \\
\text { mayor productividad. }\end{array}$ \\
\hline
\end{tabular}

Fuente: Rodríguez F, E (2006)

Según estas funciones el Terapeuta Ocupacional inicia su rol desde que el trabajador es candidato para la empresa, hasta su desempeño en la misma, razones por las cuales hace uso de diferentes herramientas, según el proceso en el que se encuentre desempeñando como profesional en formación o profesional contratado laboralmente. Entre estas vale la pena resaltar las siguientes actividades y herramientas utilizadas que de por sí, sirvieron de fundamento para llevar a cabo este proyecto como propuesta inicial y posteriormente en la implementación.

- Selección del personal: aporta al equipo los perfiles ocupacionales y profesionales del puesto de trabajo y del trabajador implicados en el análisis ocupacional. Elementos básicos para una selección armónica en cuanto a la relación trabajador puesto de trabajo - ambiente, aspecto esencial pero no único de la prevención en salud Ocupacional, puesto que conecta las evaluaciones del trabajo con las capacidades mentales, emocionales y físicas del trabajador, así como con el ambiente físico y social en el lugar de trabajo. Aquí las herramientas a utilizar son: 
Análisis Ocupacional: El análisis ocupacional en general aporta a las siguientes categorías: clasificación de la empresa y del puesto de trabajo, tareas y operaciones realizadas por el trabajador (qué hace, cómo lo hace, por qué lo hace), factores técnicos y ambientales, características requeridas por el trabajador para un desempeño satisfactorio.

Perfil ocupacional: Constituye una forma de presentación de la información relativa a las ocupaciones. Su característica principal consiste en la valoración de los factores que componen una ocupación, con el objeto de representarlos bajo la forma de un perfil el cual representa claramente el grado de dificultad o complejidad de la ocupación.”’

Con el objetivo de valorar los distintos factores de la ocupación analizada, se utiliza la información contenida en los formularios de análisis en relación con los puestos de trabajo pertenecientes a la ocupación. Como escalas de valoración es posible utilizar taxonomías en las que cada grado de dificultad contiene a los que lo preceden en orden de complejidad creciente, grados de frecuencia con que ocurren las condiciones de trabajo características de las ocupaciones. Así mismo, la elaboración de perfiles ocupacionales en relación a los conocimientos y habilidades practicas, mínimas y necesarias para el desempeño de una ocupación, presenta una clara aplicación en el campo de la certificación en calificaciones de la ocupación.

- Reglamento de Higiene y Seguridad Industrial: aquí los aportes de Terapia Ocupacional son: Participación en la conformación del reglamento, divulgar y promover el reconocimiento de las mismas por parte de la población trabajadora, comprometer tanto a la empresa como a los trabajadores a dar cumplimiento a las disposiciones legales que afecten a los programas de medicina preventiva y del trabajo, higiene y seguridad industrial.

- Subprograma de Higiene Industrial: en promoción, Terapia Ocupacional adopta acciones educativas que orienten sobre las relaciones entre el desempeño ocupacional y calidad de vida, y ofrezcan estrategias de conservación y mantenimiento. $\mathrm{Y}$ en prevención es disminuir o eliminar factores personales, ocupacionales y del medio ambiente que conduzcan a limitaciones o restricciones en el desempeño ocupacional.

Que los trabajadores alcancen bienestar ocupacional requiere del Terapeuta Ocupacional:

- Entender que el bienestar no se relaciona exclusivamente con aspectos individuales del trabajador si no que tiene que ver con la multiplicidad de factores personales, sociales, culturales y económicos en interacción para conocer todos estos factores el Terapeuta Ocupacional se sirve de un perfil socio demográfico.

- Comprender y orientar sobre la influencia de las ocupaciones, tanto para conservar y desarrollar fortalezas físicas, mentales, sociales y espirituales como para satisfacer necesidades humanas y para vivir en bienestar

- Proponer mecanismos tendientes a que las personas y grupos sociales analicen la calidad de su desempeño habitual e identifiquen las fuentes que de este desempeño contribuye para poner en riesgo el estilo de vida saludable y gratificante. 
- Diseñar estrategias de comportamiento dirigidos a favorecer y ampliar el bienestar y el cumplimiento de sus aspiraciones.

- Subprograma de Seguridad Industrial: en este subprograma de la salud ocupacional, así como en los anteriores, el rol a desempeñar se ha caracterizado y demanda la participación el establecimiento de hábitos de organización del puesto de trabajo, es decir, establecer u orientar un comportamiento saludable en el puesto de trabajo, respetando las normas establecidas para evitar accidentes de trabajo.

El Terapeuta Ocupacional fomenta el uso de estos elementos en actividades donde se muestre la importancia del bienestar físico, en la vida cotidiana del trabajador. Se puede enfatizar en las consecuencias familiares y sociales que tendrían la perdida total o parcial de una estructura corporal por descuido mal uso de un elemento. Otra actividad a desarrollar, es la relacionada con el levantamiento de panoramas de factores de riesgo, desde una perspectiva cualitativa, en la cual se busca proyectar actividades para mejorar y mantener la salud de los trabajadores, así como para prevenir los agentes de riesgo ergonómico, que pueden evocar una enfermedad profesional o un accidente de trabajo.

- Subprograma de Medicina Preventiva y del Trabajo: este es el subprograma que demanda mayor participación del profesional. La medicina del trabajo, en términos generales considera al hombre no solo como un ser productor, sino que tiene en cuenta su vida extralaboral considerando al trabajo, como un complejo dinámico.

Las actividades de la medicina del trabajo están dirigidas a la promoción, prevención, evaluación y control del estado de salud del trabajador en relación con los factores de riesgo a los que está expuesto. Así, el subprograma de medicina preventiva y del trabajo debe incluir:

- Evaluaciones médicas ocupacionales

- Exámenes médicos de ingreso

- Exámenes periódicos

- Examen de retiro

- Actividades de promoción y prevención en salud

En este orden de ideas, el profesional de Terapia Ocupacional se enfrenta a un complejo de situaciones que en la vida práctica demandan el uso de las herramientas propias de la disciplina, así como de aquellas que se han incorporado a la formación y que fundamentan su actuar interdisciplinario. Cuando hace uso de la ergonomía, como fue el caso de este proyecto y proceso, parte de la concepción ergonómica de involucrar todos aquellos agentes o situaciones que tienen que ver con la adecuación del trabajo, o los elementos de trabajo a la fisonomía humana. Así mismo, identifica factores de riesgo los objetos, puestos de trabajo, máquinas, equipos y herramientas cuyo peso, tamaño, forma y diseño pueden provocar sobre-esfuerzo, así como posturas y movimientos inadecuados que traen como consecuencia fatiga física y lesiones osteomusculares. 
Tales factores de riesgo musculo esquelético, osteomusculares o ergonómicos, nombres con los que se les puede identificar, se pueden resumir en: Postura y los problemas derivados de ella (postura mantenida, postura prolongada: Postura forzada y posturas antigravitacionales), fuerza, movimiento y fuentes generadoras tales como: Espacio de trabajo, planos de trabajo y zona de trabajo.

Lo anterior, puede desencadenas lesiones asociadas con la exposición a posturas, cargas y movimientos repetitivos, es funciones y estructuras corporales propias del sistema músculo esquelético, comprometiendo ligamentos, tendones, articulaciones, huesos, vasos y nervios. Entre estas lesiones se encuentran:

- Fatiga

- Lesiones por Trauma Acumulativo (LTA): Inflamación o ruptura del tendón y / o músculo del hombro; síndrome del túnel carpiano; inflamación de articulaciones del codo; lesión de meniscos; esguince del tobillo, dolor lumbar.

Lo expuesto hasta aquí, como aparte de los fundamentos teóricos tenidos en cuenta, permitieron sustentar, argumentar y socializar el proceso llevado a cabo en la industria de alimentos. A continuación, se continúa con la presentación de los apartes relacionados con la metodología, resultados y conclusiones derivadas de la intervención.

\section{METODO}

Para la ejecución del proyecto se partió de dos premisas fundamentales:

La primera, relacionada con el aspecto ético, necesario para llevar a cabo intervenciones donde participan seres vivos, entre ellos y para este caso, trabajadores y trabajadoras de las líneas seleccionadas. Para su aplicación, se contó con el diseño y aplicación de un consentimiento informado, en el cual se dio a conocer el objetivo del proyecto, actividades y herramientas a utilizar, respetándose el deseo de participar o no en el mismo. Estos consentimientos se incorporaron a la hoja de vida de cada trabajador y a la fuente documental del informe final.

Lo anterior, se complementó, con el consentimiento informado por parte de los representantes de la empresa, en este caso, división de seguridad y salud ocupacional, quienes solo autorizaron el registro fotográfico de algunas tareas, dada la confidencialidad de los procesos productivos. Este aspecto fue respetado por la tutora, pasantes y proyecto.

El segundo fundamento, se baso en la aplicación del Marco Lógico ${ }^{10}$, que como fundamento teórico desde investigación orienta los procesos de investigación formativa en la Universidad del Rosario y por tanto, facultad y programa de Terapia Ocupacional. El mismo busca, como herramienta analítica, la planificación para la gestión de proyectos orientado por objetivos e identificación de necesidades. Así, se establecieron tres 3 objetivos. Uno general planteado como: aplicar los procedimientos del Sistema de Vigilancia Epidemiológica ${ }^{11}$ para riesgo osteo-muscular ya diseñados en ocho líneas productivas de la planta de Alimentos y dos específicos, relacionados con: 1) Implementar un Sistema de Vigilancia Epidemiológica para riesgos osteo-musculares, mediante la evaluación y diagnostico de los trabajadores y las líneas de producción y 2) Llevar a cabo el seguimiento y control del Sistema de Vigilancia Epidemiológica para riesgos osteomusculares. 
Lo anterior y con base en Marco Lógico partió de la identificación de dos necesidades centrales, las cuales fueron respondidas mediante la implementación del Sistema de Vigilancia Epidemiológica en ocho líneas de producción:

- Primera línea (papas)

- $\quad$ segunda línea (papas y detodito),

- cuarta línea (Choquito limón),

- quinta línea (trisitos y chetos),

- séptima línea (chetos),

- octava línea (boliqueso y cheesetris),

- novena línea (gudis y chokis),

- décima línea (maní salado y manimoto) y la bodega, de la empresa.

Cada una de estas líneas, fue diagnosticada respecto a su población trabajadora, con sus puestos de trabajo mediante la aplicación de 5 instrumentos diseñados o adaptados según el caso por anteriores pasantes del Programa de Terapia Ocupacional, en la misma empresa, pero en diferentes plantas. Relacionado con esto, las líneas 3 y 6 no fueron intervenidas en este proyecto, dado que hicieron parte de una primera experiencia llevada a cabo por dos estudiantes, pertenecientes al mismo programa en el 2007 y de cuyos resultados se derivo esta segunda intervención. A continuación, se hace una breve descripción de los instrumentos:

\section{Matriz socio-Demográfica ${ }^{12}$}

Esta matriz está constituida por 23 variables de las cuales se utilizaron 18, decisión fundamentada en la orientación recibida por parte de la tutora para efectos de socialización grupal y presentación de multimedia ante toda la comunidad rosarista. No obstante, el conjunto total de información incorporada en una base de datos diseñada, se socializó, validó y entregó al sistema de información interno de la empresa, quien en la actualidad adelanta desde la dirección de salud ocupacional la supervisión al proceso nacional de control de factores de riesgo. Así, las variables analizadas se relacionaron entre otras con: nombre del trabajador o trabajadora, edad, cargo, estado civil, número de personas a cargo, nivel de escolaridad, tiempo libre, antigüedad en la empresa, antigüedad en el cargo actual, tipo de contratación, practica de actividades de salud en la empresa, diagnostico de enfermedad y condiciones del puesto de trabajo.

El desarrollo de esta matriz fue efectuado mediante la aplicación de una encuesta; en este caso, estudiantes pasantes, quienes mediante contacto personal con cada uno de los operarios en su lugar de trabajo facilitaron la orientación respectiva para responder las 18 preguntas consideradas como pertinentes en la matriz.

\section{Auto reportes de condiciones de salud ${ }^{13}$}

Consta de 4 preguntas, cada una con diferentes variables referentes a dolor, dificultades para mover estructuras corporales y sintomatología en diversas partes del cuerpo, por medio de este instrumento, se logro identificar la presencia de posibles riesgos osteo musculares a los que están expuestos los trabajadores. En este sentido, se tuvieron en cuenta los aportes generados en Colombia de las Guías Integrales ${ }^{14},{ }^{15}$ 
Los auto-reportes de condiciones de salud fueron diligenciados por los trabajadores individualmente, con la colaboración esporádica de los estudiantes pasantes para verificar la solución completa y la resolución de dudas en cuanto a los ítems necesarios.

\section{Auto-reportes de condiciones de trabajo ${ }^{16}$}

Este instrumento, es la combinación de dos auto-reportes, los cuales fueron obtenidos de las investigaciones de sistemas de vigilancia epidemiológica de factores de riesgo, cargas y posturas y de investigaciones para la prevención de lesiones de columna vertebral y miembros superiores causadas por riesgo ergonómico ${ }^{17181920}$. El objetivo principal del mismo, consiste en combinar los auto-reportes para identificar carga física, posturas y deficiencias que presenta el ambiente laboral.

Los auto-reportes de condiciones de trabajo, fueron diligenciados por los trabajadores de manera individual, con la colaboración esporádica de los estudiantes pasantes para verificar la solución completa y la resolución de dudas en cuanto a los ítems necesarios.

\section{Análisis ocupacional ${ }^{21}$}

El análisis ocupacional es una metodología utilizada para la identificación de los diferentes comportamientos laborales correlacionados con las distintas tareas y ocupaciones ejecutadas $^{22}$; a su vez, es un instrumento diagnostico que mide el nivel de dominio en su desempeño laboral. El mismo, identifica el conjunto de comportamientos laborales básicos requeridos en los trabajadores que son comunes en las distintas áreas ocupacionales. Su finalidad, consiste en: evaluar y describir el trabajo; evaluar y describir la ejecución de las diferentes tareas por parte del trabajador y emitir recomendaciones relacionadas con higiene postural, seguridad industrial y promoción y prevención.

El análisis ocupacional se desarrollo mediante la observación directa del puesto de trabajo durante la ejecución de las diferentes tareas, este es dependiente en su totalidad de la observación del Terapeuta Ocupacional.

\section{Descripción de la herramienta evaluativa $\mathrm{OWAS}^{23}$}

Es una herramienta sencilla y útil destinado al análisis ergonómico de la carga postural. Su aplicación, proporciona buenos resultados, tanto en la mejora de la comodidad de los puestos, como en el aumento de la calidad de la producción, consecuencia ésta última de las mejoras aplicadas. La misma, referencia tres aspectos relacionados con la posición de la espalda, los brazos y las piernas en donde el evaluador durante el análisis de cada una de las tareas debe escoger entre las opciones dadas para cada posición.

Esta herramienta ergonómica, se aplicó mediante la observación directa de los puestos de trabajo durante la ejecución de las diferentes tareas. La misma, es dependiente en su totalidad de la observación del terapeuta ocupacional.

\section{RESULTADOS}

A continuación se exponen los resultados más representativos según los instrumentos descritos anteriormente. 
1. Matriz socio-demográfica: La matriz fue aplicada a manera de encuesta a 200 trabajadores de la planta de alimentos. Los resultados más relevantes se dieron respecto a las variables de: edad, sexo, nivel de escolaridad y hábitos saludables y no saludables de los trabajadores y algunas molestias dentro del sitio de trabajo.

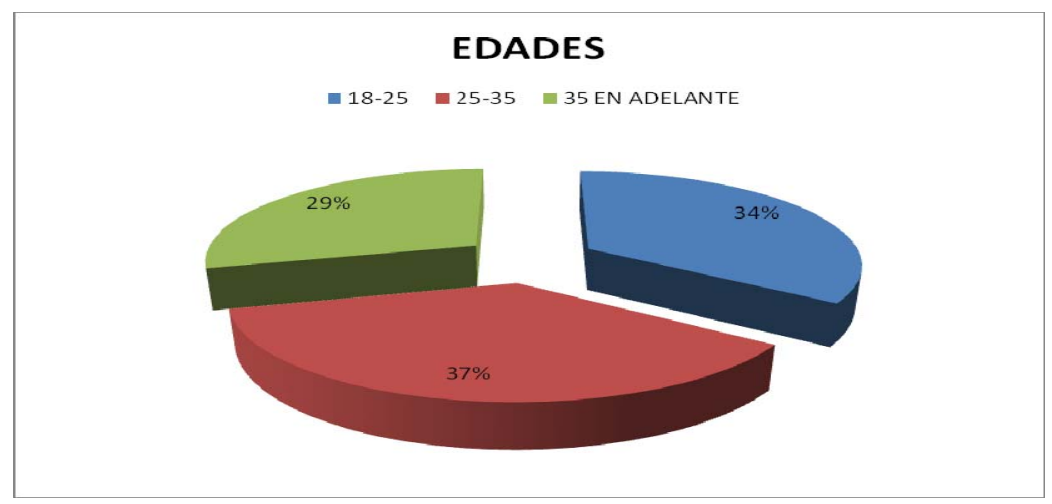

\section{Gráfica No. 1}

En los rangos de edad se encontró que un 37\% de la población de la planta se encuentra entre los 25 a 35 años; en la cual según el desarrollo de las habilidades ocupacionales de Mosey, citado por Romero ${ }^{24}$, ya existe una percepción de autocontrol, se presenta una adecuada participación en grupos y las personas se reconocen como seres productivos que constituye un sistema social. (Ver Gráfica No. 1)

\section{Gráfica No. 2}

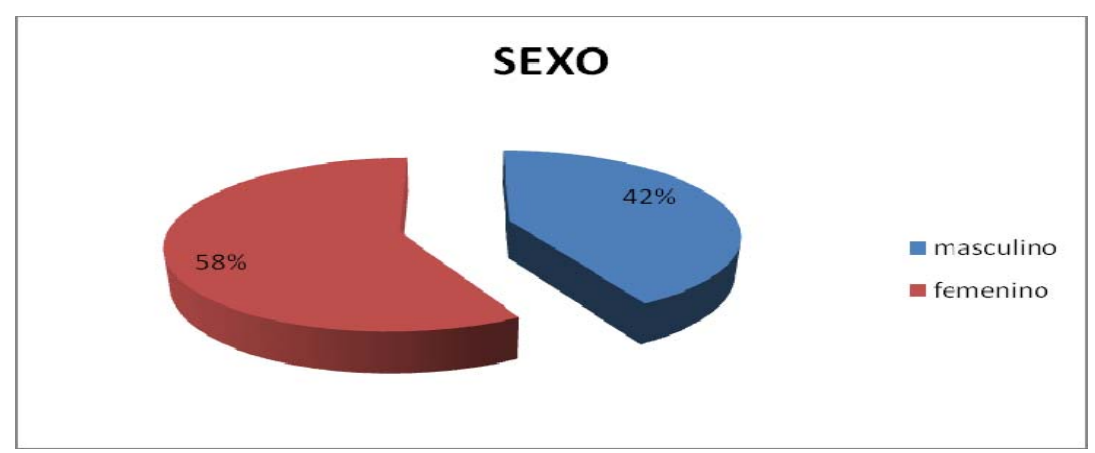

Respecto a esta variable, se encontró que un 58\% de la población son mujeres y el $42 \%$ restante son hombres. La mayoría de la población operaria de las nueve líneas de producción es de sexo femenino, esto se podría deber a la creencia cultural respecto a la cual, las mujeres son más rentables, al presentar mayor capacidad para relacionarse, realizar los trabajos de forma ordenada y realizar con mayor facilidad tareas de precisión. (Ver Gráfica No. 2) 


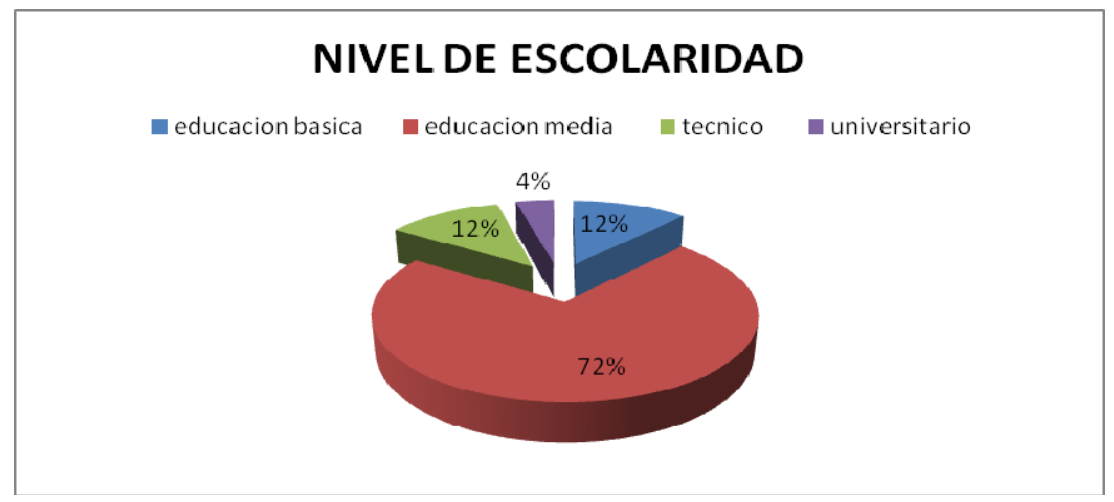

Gráfica No. 3

Se encontró que el 72\% tiene educación tiene una educación básica, lo cual según la Clasificación Nacional de las Ocupaciones ${ }^{25}$, aspecto considerado para esta variable como adecuado según nivel de calificación por área de desempeño. (Ver Gráfica No. 3)

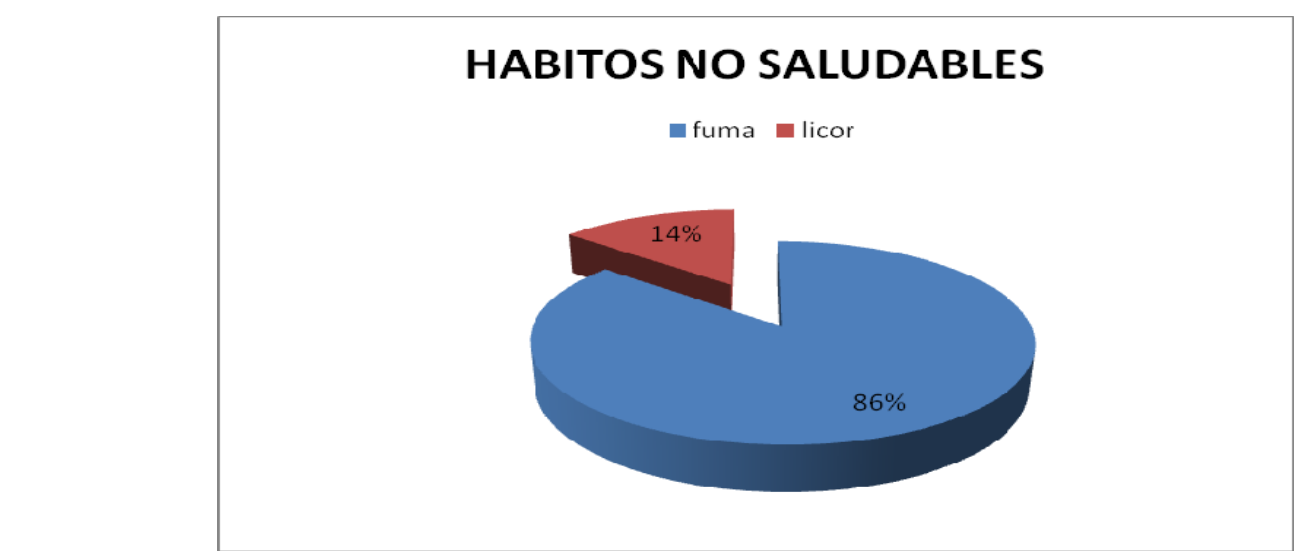

Gráfica No.4

Para esta variable, se encontró que la mayoría de los trabajadores son fumadores y un porcentaje muy corto son tomadores ocasionales, aspectos que según la legislación nacional han de ser intervenidos desde los programas de salud ocupacional. )Ver Gráfica No. 4)

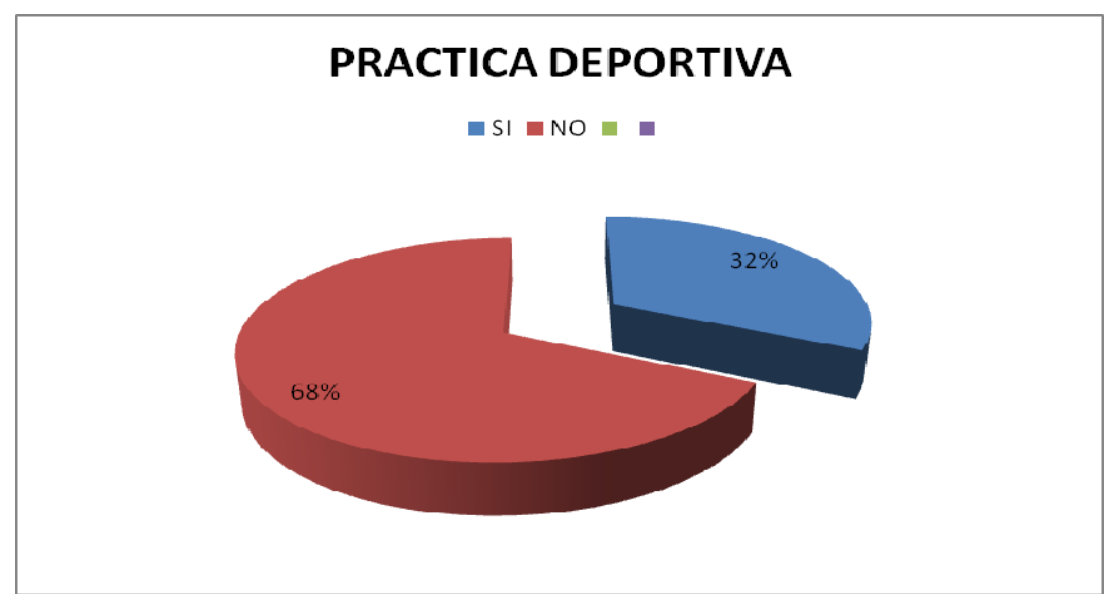




\section{Gráfica No. 5}

En referencia a la práctica de deportes el 68\% respondieron que no realizan ningún tipo de actividad; respecto a esto, se cree que es de gran importancia la realización de ejercicio al permitir una vida más sana y saludable. As mismo, este tipo de actividades se incluyen actualmente en el subprograma de medicina preventiva y del trabajo. (Ver Gráfica No. 5)

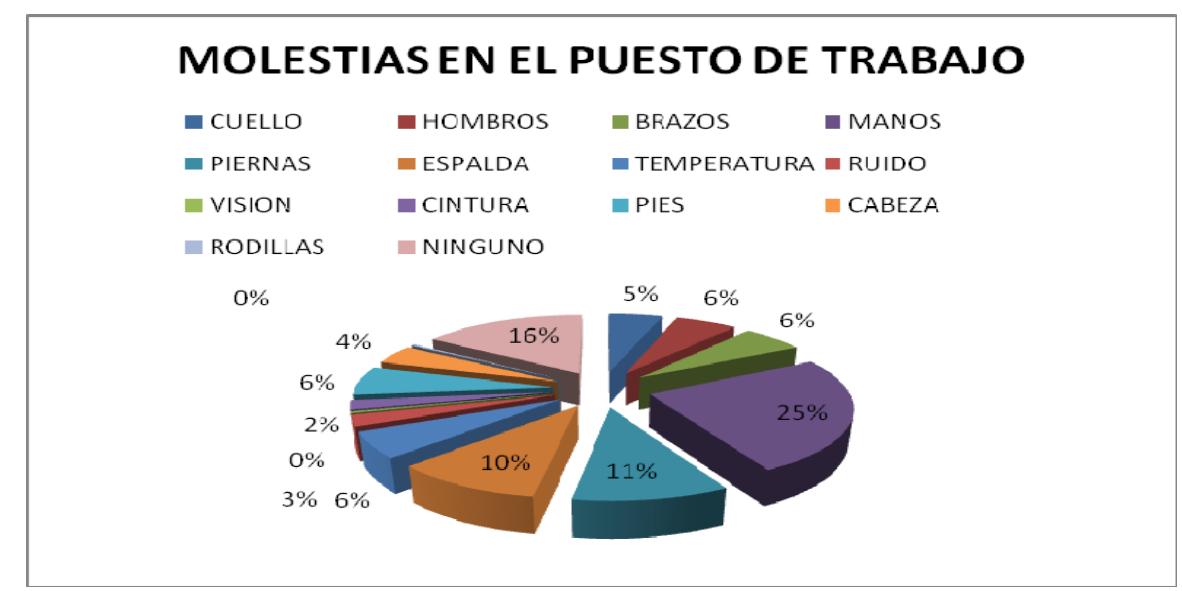

\section{Gráfica No. 6}

En referencia a las molestias en el puesto de trabajo se encontró que un 25\% refirió dolor en cintura, dato que puede estar asociado a los prolongados periodos de tiempo que los operarios asumen la posición bípeda. El dato menos relevante se dio a nivel dio a nivel de dolor de hombros, con un 2\%. (Ver Gráfica No. 6)

2. Auto-reporte de Condiciones de Salud: Los resultados obtenidos por medio de la aplicación de este instrumento, fueron divididos por actividades en la planta. La división se caracterizo en 4 acciones: a) Empaque, b) Canasta, c) Cendis y d) Mantenimiento.

Las graficas expuestas a continuación son las de la actividad de Empaque, debido a que el mayor número de trabajadores en la planta se desempeña en esta actividad.

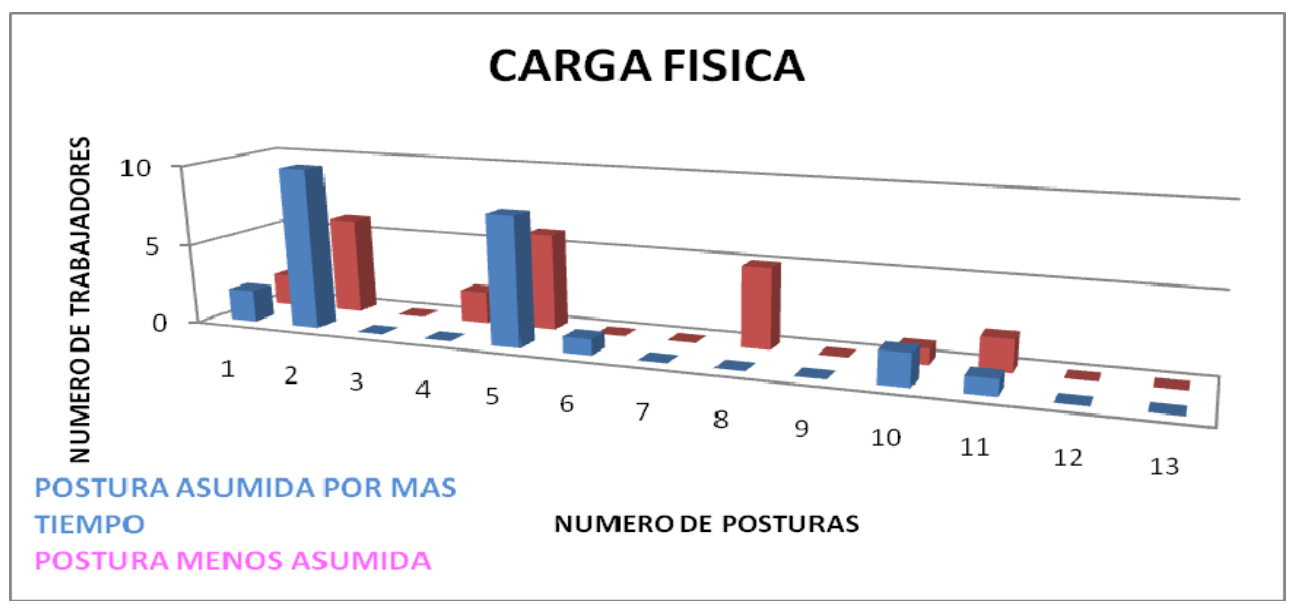




\section{Gráfica No. 7}

Respecto a esto, la postura asumida por mayor tiempo durante la jornada laboral es la segunda (bípedo, espalda recta, con una de las dos piernas con flexión de rodilla de $40^{\circ}$ y los miembros superiores flectados, hombro $45^{\circ}$, codo $20^{\circ}$ ), seguida de la quinta ( bípedo, con una de las dos piernas con flexión de rodilla de $40^{\circ}$, y cintura pélvica con $30^{\circ}$ en flexión) y la postura menos asumida fue la 13 (de rodillas, espalda recta y con y los miembros superiores flectados, hombro $45^{\circ}$, codo $20^{\circ}$ y flexión de muñeca de $90^{\circ}$ ). (Ver Gráfica No. 7)

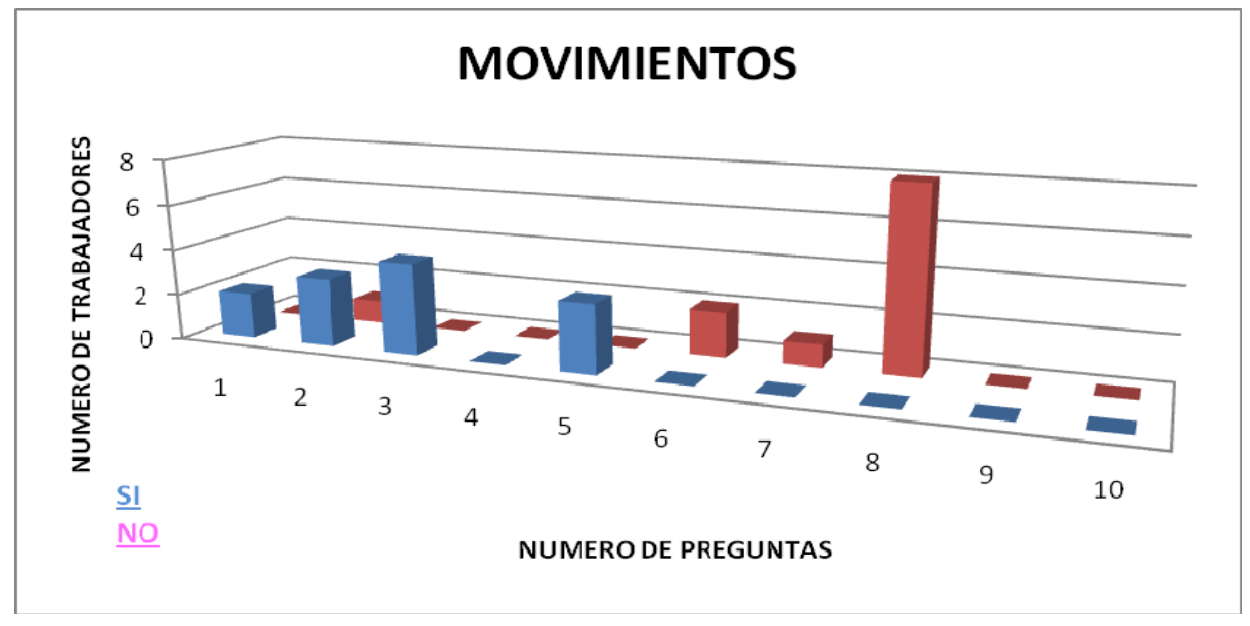

\section{Grafica no. 8}

Las tareas con mayor presencia de realización, fueron las de larga duración (tareas en las que las operaciones son repetitivas y duran mas del 80\% de la ejecución total de la tarea.), seguidas del trabajo que implica movimientos de cuello y espalda. Las tareas menos realizadas son aquellas que requieren desplazamientos con transporte de cargas mayores a tres metros, seguido de la manipulación de materiales con peso de 5 kilogramos o más. (Ver Gráfica No. 8)

Relacionado con lo anterior, las siguientes graficas evidencian las acciones efectuadas por los trabajadores durante las diferentes tareas en el lugar de trabajo. Las acciones se entienden aquí, como el conjunto de exigencias motoras gruesas para llevar a cabo una acción.

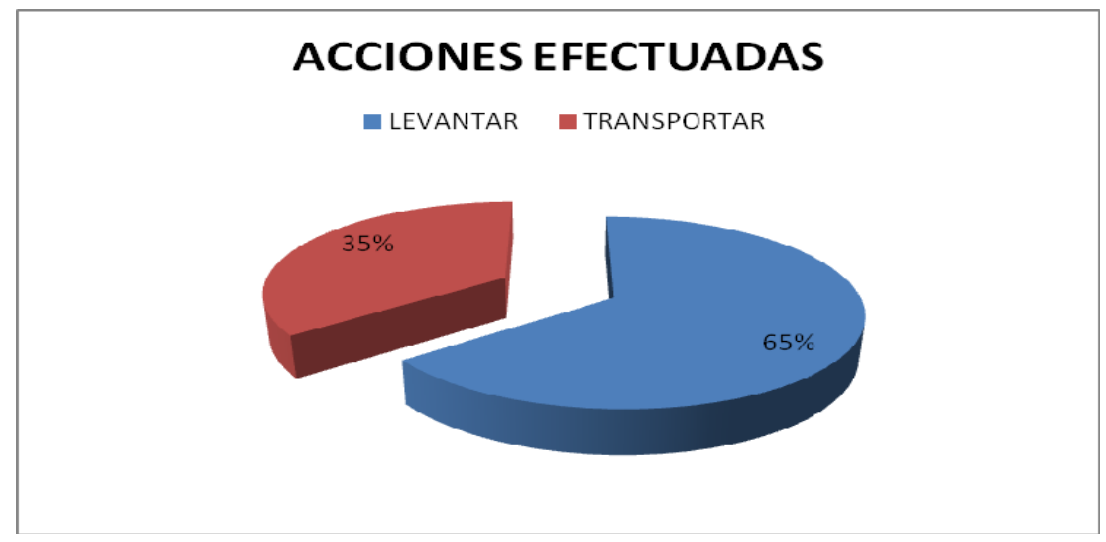




\section{Gráfica No. 9}

La acción mas realizada en cuanto a manipulación de cargas es la de levantar con un 65 \%, seguida de transportar con un 35\%. Relacionado con esto, la forma en que se manipulan las cargas resultó ser individual en un 96\% y colectiva en un 4\%. (Ver Gráfica No. 9). Esta información, se complementó con la indagación respecto al mecanismo utilizado para la manipulación de las cargas, encontrándose que un 88\% de los trabajadores manipula de forma manual, un 8\% de forma automática y un 4\% de forma mecánica.

\section{Gráfica No. 10}

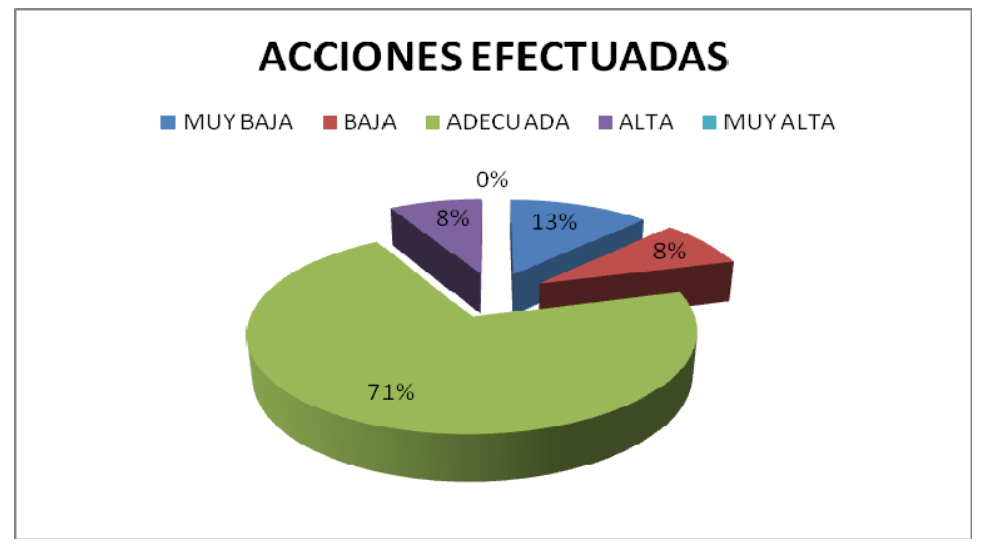

En cuanto a cómo encuentran los trabajadores la altura del plano de trabajo para el desarrollo de las tareas, se encontró que un 71\% las consideró como adecuadas; un 13\% las consideró como muy altas, un 8\%, las consideró como altas y el 8\% restante como bajas. (Ver Gráfica No. 10). Estos datos, se complementaron con el diagnóstico establecido respecto a las características del área de trabajo, aspecto respecto al cual los trabajadores que las condiciones de iluminación eran adecuadas y manifestaciones de incomodidad respecto a la temperatura (el calor es molesto).

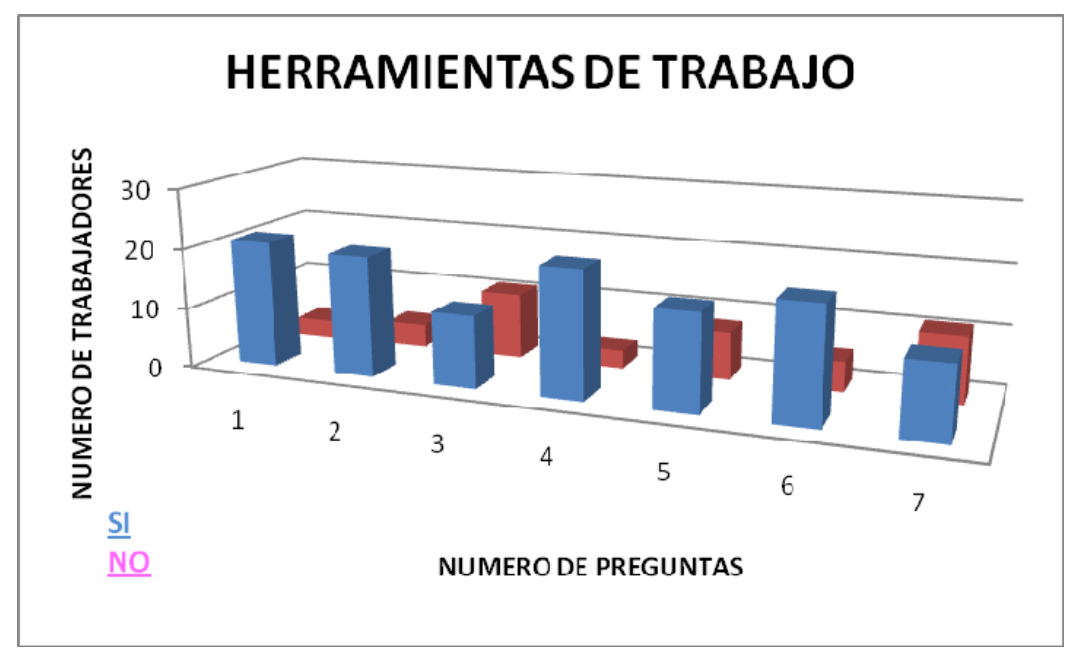

Gráfica No. 11

Otro aspecto diagnosticado, fue el relacionado con las características de las herramientas y maquinas de trabajo, respecto a lo cual 22 trabajadores las refirieron 
como indicadas y en buenas condiciones. Como dato significativo de forma negativa, la mayoría de los trabajadores, refirió que estas no son suficientes, seguido de la percepción de peligro en su uso. (Ver Gráfica No. 11) Estos hallazgos, fueron complementados con el diagnostico respecto al nivel de esfuerzo requerido para la ejecución de las actividades, encontrándose que para un $76 \%$ de la población resultó ser medio, pesado para un $16 \%$ pesado y liviano para un $8 \%$.

Otro aspecto evaluado, se relacionado con la exploración de signos y síntomas relacionados con la presencia de dolor, aspecto frente al cual la siguiente Gráfica expresa los resultados encontrados.

\section{DOLOR}

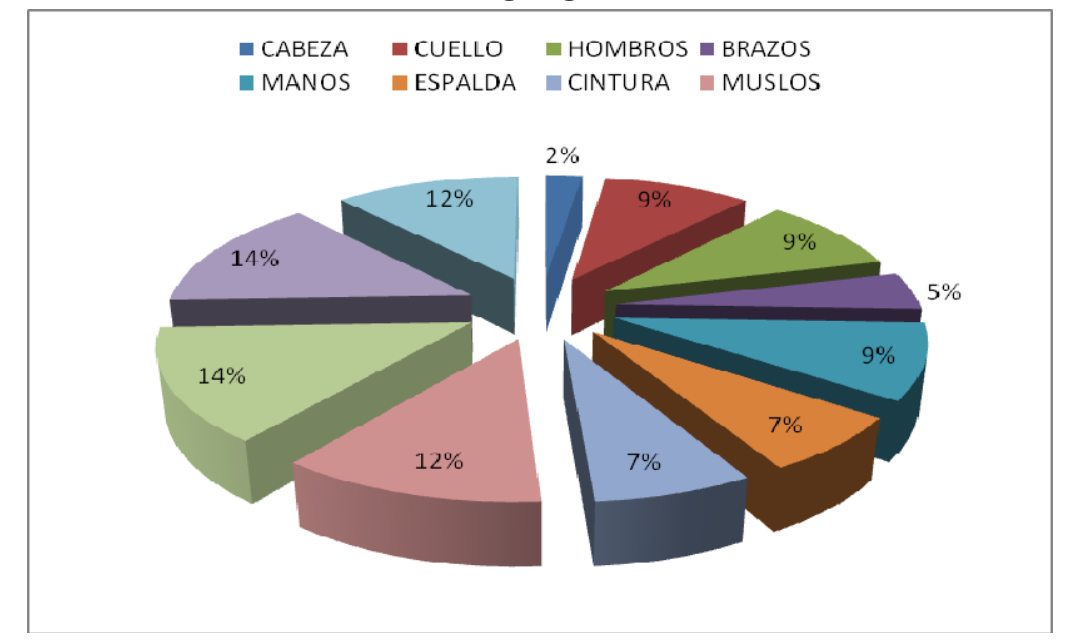

Gráfica No. 12

Los síntomas de dolor presentados durante la jornada laboral, se concentraron específicamente en: dolor en la cintura, en hombros y en muslos y los dolores que menos se presentan según referencia de los trabajadores fueron en: cabeza, seguido de brazos. (Ver Gráfica No. 12). Estos hallazgos se complementaron con la exploración respecto a las dificultades presentes durante la jornada laboral, relacionadas directamente con los signos y síntomas referidos. Así, se encontró que en un $21 \%$ las mayores dificultades se dieron para la movilidad de cuello, 17\%, disminución en el movimiento de los brazos, en un $17 \%$ dificultad para mover las piernas, en un $16 \%$ dificultad en el movimiento de las manos, $15 \%$ dificultad en la velocidad de los movimientos y en un $14 \%$ dificultad para mover el cuello. Esto se complementó con el diagnóstico cualitativo respecto a la sensación percibida por los trabajadores, de estos signos y síntomas. En este sentido, se encontró que un 19\% refirió sensación de adormecimiento; un 18\% de inflamación; un 18\% de ardor, un 16\% sensación de frío y calor y un 13\% refirió sensación de fatiga. (Ver Gráfica No. 13) 
$\because$ FRIO $\square$ CALOR $=$ ADORMECIMIENTO $\square$ INPLAMACION $=$ ARDOR $=$ FATIGA
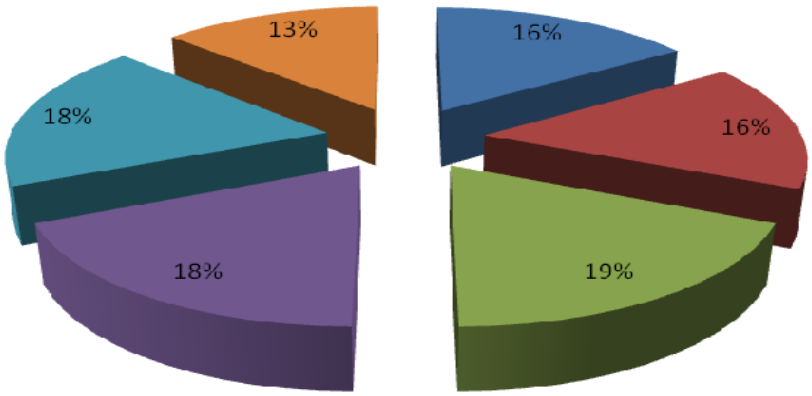

\section{Gráfica No. 13}

3. Análisis ocupacionales: La emisión de los resultados de análisis ocupacionales, demandó la elaboración y propuesta a la empresa de un semáforo de riesgos. En este, se ubican de manera estratégica todos los puestos de trabajo, dependiendo el riesgo al que se encuentren expuestos; la caracterización de los diferentes puestos de trabajo se realizó teniendo en cuenta, que los ubicados en el semáforo verde no requieren cambios; los ubicados en el semáforo amarillo requieren cambios a mediano plazo; y los ubicados en el semáforo rojo requieren cambios inmediatos. A continuación, se presenta el esquema propuesto desde proyecto como uno de los resultados centrales del mismo. Nótese, que 4 puestos de trabajo se ubicaron en semáforo amarillo y 5 en rojo. (Ver esquema No. 1)

\section{SEMAFORO}

\section{VERDE}

\section{SEMAFORO}

\section{AMARILLO}

\section{LINEA}

1-2-4-5-7-8

9

9

1-2-4-5-7-8.

\section{PUESTO DE TRABAJO}

EMBOLSADO

EMPAQUE CHOKIS

PREPARACIONGUDIS

SELLADO

\section{ROJO}


LINEA

10

1-2-4-5-7-8.

1-2-4-5-7-8.

9
PUESTO DE TRABAJO

PREPARACION MANI SALADO

Y MANI-MOTO

CANASTERO

ESTIBADO

PREPARACION CHOKIS

Esquema No. 1 Propuesta semaforiazción de puestos de trabajo desde la perspectiva de Terapia Ocupacional.

\section{DISCUSIÓN}

De acuerdo a las evaluaciones aplicadas en la industria de alimentos, se elaboró una semaforización de riesgos osteo-musculares, en donde se encontró que los puestos de trabajo que requieren cambios inmediatos son: preparación de maní salado y manimoto, preparación chokis, canastero y estibado, siendo estos aquellos puestos en los que se realizan levantamientos y transporte de cargas (análisis ocupacional OWAS). Los puestos de trabajo que se ubicaron en el semáforo en color amarillo, haciéndose necesarios cambios a mediano plazo, fueron: embolsado, empaque chokis, preparación Gudis y sellado. Estos puestos de trabajo, tienen en común la presencia de movimientos repetitivos.

Del mismo modo, la elaboración de un semáforo de riesgos osteo-musculares, como propuesta novedosa desde Terapia Ocupacional para la industria de alimentos, se convierte en una herramienta sintetizada, de fácil manejo para la identificación de puestos de trabajo en alto, medio y bajo riesgo y su aplicabilidad puede llegarse a dar para todas las plantas presentes en Colombia, sobre todo si se tiene en cuenta que como herramienta puede hacer parte de los Sistemas de Vigilancia Epidemiológica diseñados e implementados por las industrias colombianas, las cuales haciendo uso de la salud ocupacional buscar contar con programas útiles, encaminados a promover la salud, el bienestar físico y emocional y a prevenir los accidentes de trabajo, las enfermedades profesionales, los ausentismos, las incapacidades y por tanto, el deterioro en el desempeño ocupacional de la población trabajadora. Estos aspectos, lograron intervenirse mediante este proyecto que contó con una modalidad complementaria que garantizó el cumplimiento de las recomendaciones dadas a la empresa. Esta modalidad, se identifica como Práctica de Profundización del Programa de Terapia Ocupacional, experiencia que buscó dar continuidad a las acciones propuestas y fortalecer las competencias adquiridas en el sector trabajo, como área de ejercicio profesional en Colombia.

De igual modo, los resultados obtenidos y expuestos a lo largo de este artículo y que hacen parte de un documento final, más extenso, entregado a la empresa respectiva, no solo evidenciaron un diagnostico y una caracterización, también permitieron dar a conocer las dificultades que existen cuando se desean implementar procesos de formación en autocuidado para los adultos, siendo este el principal reto de Terapia Ocupacional cuando hace intervención en empresas. En suma, los aportes generados son actualmente motivo y 
punto de partida al interior del programa académico para futuros estudiantes, que interesados por desarrollar sus procesos de investigación formativa y de profundización ya plantean replicar estoas acciones en otras plantas de la misma industria, en otros procesos productivos y con otras poblaciones.

Para finalizar, Terapia Ocupacional pudo incursionar en forma planeada y coordinada, identificando características, exigencias y requerimientos de la empresa y de su población, con una búsqueda constante de favorecer el desempeño productivo y competente mediante acciones tales como promoción ocupacional, prevención de riesgos ocupacionales, formación de los trabajadores y propuestas novedosas a la empresa. Igualmente, participó en el análisis de puestos de trabajo, mediante el uso de herramientas disciplinares e interdisciplinares en enmarco del programa de salud ocupacional de la industria de alimentos seleccionada para este proyecto. 


\section{REFERENCIAS BIBLIOGRÁFICAS}

${ }^{1}$ POLO J. Retribución Emocional, Segunda Edición. Editorial Granica. España. 2005. Pág. 13-17

${ }^{2}$ MINISTERIO DE TRABAJO Y SEGURIDAD SOCIAL. Decreto 614. Colombia. 1984

${ }^{3}$ MINISTERIO DE TRABAJO Y SEGURIDAD SOCIAL. Resolución 1016. Colombia .1989

${ }^{4}$ CONGRESO DE LA REPUBLICA DE COLOMBIA. LEY 100. Colombia. 1993

${ }^{5}$ MINISTERIO DE LA PROTECCION SOCIAL. Decreto 1295 de 1994, reformado por Ley 776. Colombia. 2002

${ }^{6}$ TRUJILLO, Alicia. Terapia Ocupacional, conocimiento y práctica en Colombia. Primera Edición, Editorial Universidad Nacional de Colombia; 2002. Pág. 66 - 69.

${ }^{7}$ RODRIGUEZ F. Papel del Terapeuta Ocupacional en Salud Ocupacional y Ergonomía. Documento inédito elaborado como guía para el programa académico de campo. Programa de Terapia Ocupacional. Facultad de Rehabilitación y Desarrollo Humano, Universidad del Rosario. Colombia. 2006

${ }^{8}$ RODRIGUEZ F. Papel del Terapeuta Ocupacional en Salud Ocupacional y Ergonomía. Documento inédito elaborado como guía para el programa académico de campo. Programa de Terapia Ocupacional. Facultad de Rehabilitación y Desarrollo Humano, Universidad del Rosario. Colombia. 2006. Pág. 15-20

${ }^{9}$ RODRIGUEZ F.(2006) opus cit. Pág. 25-40

${ }^{10}$ RIOS, A. Documento de Investigación Formativa para la facultad de Rehabilitación y Desarrollo Humano, Programa de Terapia Ocupacional. Universidad del Rosario. Colombia. 2007

${ }^{11}$ ROBAYO L, VICTORIA D. Informe Final proyecto: "Sistema de Vigilancia Epidemiológica para una industria de Alimentos, planta la Marqueza en Bogotá”. Colombia. Universidad del Rosario. 2008. Pág 10-15

${ }^{12}$ ROBAYO l. (2008) opus cit. Pág.15-20

${ }^{13}$ ROBAYO l. (2008) opus cit. Pág. 23-25

${ }^{14}$ MINISTERIO DE LA PROTECCION SOCIAL. Guías de atención Integral basada en la evidencia para desordenes musculo esqueléticos (DME) relacionados con movimientos repetitivos de miembros superiores (síndrome de túnel de carpiano, epicondilitis y enfermedad de De Quervain. (GATI-DME). Colombia. 2006

${ }^{15}$ MINISTERIO DE LA PROTECCION SOCIAL. Guías de atención Integral basada en la evidencia para Dolor Lumbar. (GATI-DL). Colombia. 2006

${ }^{16}$ ROBAYO l. (2008) opus cit. Pág. 35-45

${ }^{17}$ ESTRADA, J. La ergonomía y el control de accidentes de trabajo y enfermedad profesional. Colombia, ISSN: , 2002 vol:48 fasc: 282 pág: 41 - 45

${ }^{18}$ ESTRADA, J. Sistema de vigilancia epidemiológica para la prevención de los desórdenes acumulativos traumáticos. Colombia, Revista Facultad Nacional De Salud Pública ISSN: 0120-386X, 2000 vol:17-18 fasc. 02-01 Pág: 95 - 123

19 MINISTERIO DE LA PORTECCION OSCIAL. Plan Nacional de Salud Ocupacional 2003-2007. Colombia

${ }^{20}$ MINISTERIO DE MINAS Y ENERGIA, EMPRESA COLOMBIANA DE PETRÓLEOS ECOPETROL.

Sistema de Vigilancia Epidemiológica Para La Prevención de Los desordenes Acumulativos traumáticos en la Gerencia De Barrancabermeja de Ecopetrol. República de Colombia. Colombia. 1994

${ }^{21}$ PUJOL J. Análisis Ocupacional. Manual de aplicación para instituciones de formación, Montevideo, Cinterfor/OIT, 1980

${ }^{22}$ RODRIGUEZ, E. Documento guía para la asignatura de Análisis Ocupacional y ergonomía. Documento inédito. Facultad de Rehabilitación y Desarrollo Humano. Programa de Terapia Ocupacional. Colombia. 2007

${ }^{23}$.http://www.ergonautas.upv.es/. Universidad Politécnica de Valencia. Fecha de consulta Febrero de 2009.

${ }^{24}$ ROMERO D. Terapia Ocupacional Teoría y Técnicas. Primera edición. Editorial Elsevier, España, 2008

${ }^{25}$ División de Estudios Ocupacionales Dirección de Empleo SENA, Dirección General. Clasificación Nacional de Ocupaciones C.N.O. Una herramienta para gestión de empleo y formación. 1996. 